\title{
CARRIER-MEDIATED HEAT TRANSPORT
}

\author{
J. D. GODDARD, J. S. SCHULTZ and S. R. SUCHDEO \\ Department of Chemical Engineering, University of Michigan, Ann Arbor, Michigan 48104, U.S.A.
}

(Received 12 December 1973; accepted 7 April 1974)

\begin{abstract}
It is shown how an asymptotic, boundary-layer method, developed specifically for application to facilitated mass transport, can also be applied to the corresponding problem of facilitated enthalpy transport. The method, which merely entails an implicit algebraic computation, is applied to the (hydrodynamic) film-theory model for heat transfer between a hot or cold wall and a reversibly dissociating gas mixture, $2 \mathrm{NO}_{2} \rightleftarrows 2 \mathrm{NO}+\mathrm{O}_{2}$, which has previously been treated by Brian and Bodman [1]. Good agreement is found with their approximate analytical formula over the parameter range of their computations. However, for more extreme states of dissociation and larger overall temperature differences, the present results differ from those given by their approximate formula, especially for the cooling (cold wall) problem.
\end{abstract}

\section{INTRODUCTION}

The conduction of heat in chemically reactive systems is by no means a novel topic in the general area of diffusional transport phenomena. It has been the subject of several previous works, which are exemplified most notably by those of Hirshfelder[2], Brian and Reid[3], Brian and Bodman [1], Bodman et al. [4], Fan and Mason [5], and co-workers. One of the principal objectives of such studies has been to predict theoretically the reactive enhancement of steady-state heat transfer through a chemical mixture due to reversible thermal dissociation and recombination accompanied by molecular diffusion of various chemical species. If, in such systems, say reacting gas mixtures, the intrinsic rates of the chemical reactions are, in some appropriate sense, small compared to diffusional transport rates then one obtains the so-called 'frozen' (conductivity) regime, where reaction exerts a negligible influence on energy transport. At the opposite extreme, where reaction rates are in effect infinitely rapid, one obtains the (reaction) 'equilibrium' regime and, usually, maximum enhancement of heat flux.

In either of these asymptotic regimes one is generally able to perform fairly direct computations of the heat flux through a mixture from given transport and equilibrium properties; whereas, otherwise, one has to solve a set of non-linear differential equations, involving diffusion and reaction rates and governing spatial temperature and composition fields. While such boundary-value problems are in principle straightforward, albeit not analytically tractable, and actual numerical computation can be difficult and time-consuming, even with advanced computer-executed numerical schemes. These practical difficulties are traceable directly to a boundary layer structure that develops whenever reaction rates are moderately to highly rapid.

This boundary-layer nature of the nearequilibrium regime was anticipated in the early works of Hirshfelder [2], but, up to the present, no general mathematical techniques have apparently been proposed to cope with the problem in this particular physical context. However, in very recent times there has been a good deal of effort devoted to the treatment of problems closely akin to the above in the related area of facilitated mass transport.

Although there had been a previous longstanding interest in the reactive enhancement of mass transport, such problems have received renewed attention because of their possible relevance to carrier-mediated biological transport in membranes and liquid films. Thus, Goddard et al.[5] have given boundary-layer analyses of the nearequilibrium regime in this context, while Kreuzer and Hoofd [6] and Smith et al. [7] have proposed an approximate boundary-layer method which appears to give very accurate results, even far from the equilibrium regime and well into the near-diffusion or frozen regime [8].

The purpose of the present work is to consider an illustrative application of the above boundary-layer method, involving heat conduction through a twocomponent reactive gas layer to an equilibrium 
mixture of the gases, a problem which has been previously considered by Brian and Bodman [1] $\dagger$ as a film-theory model for convective heat transfer from a wall to a flowing dissociating gas such as $\mathrm{NO}_{2}$. In their work, Brian and Bodman employed numerical solutions of the governing equations to investigate the effects of finite temperature driving force between wall and the equilibrium free-stream or bulk. They were thereby able to explore some of the limitations on a much used linearized theory, strictly applicable only to a weakly perturbed film with vanishing thermal driving force [cf. refs. 8-10] as well as a modification thereof which they propose as an approximate analytical solution.

Our main objective here is to compare numerically the results of the boundary-layer method with the approximate analytical method of B \& B, for a physical system which is amenable to experimental study [4]. The boundary-layer method itself can be applied in a relatively straight-forward way to much more complex systems $[1,8]$.

\section{BASIC EQUATIONS AND SOLUTION METHOD}

Here, we consider as the model system a binary gas mixture of $A$ and $B$ undergoing a reaction

$$
2 A \rightleftarrows 3 B,
$$

with elementary (mass-action) kinetics

$$
-r_{A}=\frac{2}{3} r_{B}=k_{F} p^{2} y^{2}-k_{R} p^{3}(1-y)^{3},
$$

where $t$ is the mole fraction of $A$, hence, $1-y$ the mole fraction of $B ; k_{F}$ and $k_{R}$ are kinetic constants depending only on temperature $T$; and $p$ the pressure. Following B \& B, one can consider this to be a model for the dissociation of $\mathrm{NO}_{2}$ according to

$$
2 \mathrm{NO}_{2} \rightleftarrows 2 \mathrm{NO}+\mathrm{O}_{2},
$$

for an equilibrium bulk gas consisting of stoichiometrically and partially decomposed $\mathrm{NO}_{2}$, provided that the properties of $\mathrm{O}_{2}$, most notably its diffusivity in the mixture, are taken to be identical with those of NO. Accordingly, the degree of dissociation, defined by

$$
\xi=\frac{1-y}{1+y / 2}
$$

can serve equally well as a composition variable.

†Hereinafter referred to as "B \& B".
For the case of one-dimensional steady-state diffusion with no thermal effects of mixing, and with flux conditions $N_{A}=0, N_{B}=0$ on some impermeable wall, located say at $x=0$, the relevant species and energy balances reduce to

$$
N_{A}+\frac{2}{3} N_{B} \equiv 0
$$

and

$$
q-\underline{H}_{A} N_{A}+\underline{H}_{B} N_{B}=q+(\Delta H) N_{A} \equiv q_{w},
$$

a constant for all

$$
x \geqslant 0,
$$

as given also in B \& B, where $q_{w}$ denotes the wall heat flux and $\Delta H$ the heat of reaction.

For ideal gas mixtures, with constant pressure and negligible thermo-diffusion (or other coupling between heat and mass diffusion), the usual laws for mass flux and heat conduction give, on substitution into (5) and (9), the differential equations

$$
\begin{gathered}
\frac{\mathrm{d}}{\mathrm{d} x}\left[\left(\frac{D C}{1+y / 2}\right) \frac{\mathrm{d} y}{\mathrm{~d} x}\right]--r_{A}(y, T) \\
-k \frac{\mathrm{d} T}{\mathrm{~d} x}+\frac{\Delta H D C}{1+y / 2} \frac{\mathrm{d} y}{\mathrm{~d} x}=q_{w}
\end{gathered}
$$

which, with the expression of (2) for $r_{A}$, are those given by B \& B, whose notation we shall adopt for purposes of comparison. The boundary conditions relevant to the film theory model used by B \& B are

$$
\text { at } x=0, \quad \frac{\mathrm{d} y}{\mathrm{~d} x}=0, \quad T=T_{w}
$$

and

$$
\text { at } x=L, \quad y=y_{0}, \quad T=T_{0} .
$$

where the variables $y_{0}, T_{0}$ are assumed to obey the condition of chemical equilibrium in the bulk which, by (2), also involves an adjustable pressure p.

The relations (7) to (10) together with (2) are then to be solved in order to determine $q_{w}$, the wall heat flux in (6), given $T_{w}, T_{0}$ and $y_{0}$.

The rate constants $k_{F}, k_{R}$ in (2) are assumed to have an Arrhenius-type temperature dependence, such that the system (7) to (10) is highly non-linear both in mole fraction $y$ and temperature $T$ and generally requires numerical methods for its solution. As pointed out above, this has been carried 
out in B \& B for some special parameter values, whereas here we wish to apply an approximate boundary-layer method which requires relatively simple integrations and algebraic operations for the computation. For this purpose, it is desirable to introduce formally a somewhat arbitrary dimensionless Damköhler number, which for later convenience we take to be

$$
m=\frac{2 y_{0}\left(1+y_{0} / 2\right)^{2}}{\left(1-y_{0}\right)} \frac{k_{F 0} p^{2} L^{2}}{C_{0} D_{0}}
$$

the quantity used in B \& B as a measure of the rate of reaction relative to diffusion.

Then, as indicated schematically in Fig. 1, one assumes outer equilibrium temperature and composition fields $\hat{T}(x), \hat{y}(x)$ in a region $0\left(m^{-1 / 2}\right)<$ $x / L \leqslant 1$, which satisfy, instead of $(7)$, the condition of reaction equilibrium

$$
r_{A}(\hat{y}, \hat{T})=0
$$

together with the flux relation (8) and the (compatible) conditions (10) at $x=L$. The equilibrium profiles $\hat{y}(x), \hat{T}(x)$ are to match $x=0$ (asymp-

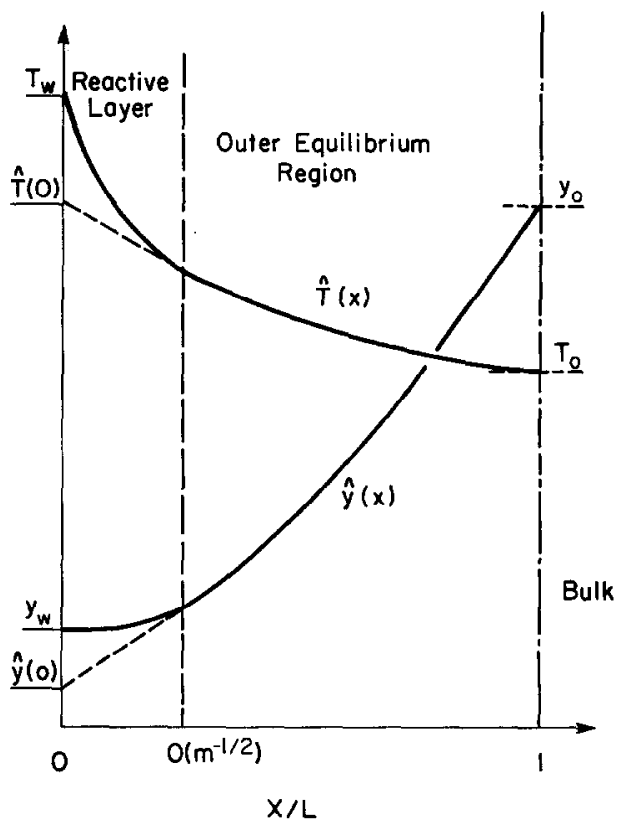

Fig. 1. Schematic profiles of temperature and concentration adjacent to a hot wall in contact with a reversibly reacting gas mixture. $T=$ true temperature, $\hat{T}=$ temperature assuming local reaction equilibrium, $y=$ mole fraction of $A, \hat{y}=$ mole fraction of $A$ assuming local equilibrium, $x / L=$ distance coordinate from the wall into the gas film. totically for large $m$ ) with boundary-layer solutions which are valid in a reactive layer $0 \leqslant x / L<$ $0\left(m^{-1 / 2}\right)$ and which are to satisfy the exact boundary conditions (9) at $x=0$.

Within the approximate boundary-layer analysis, employed by Kreuzer and Hoofd [6] one obtains the relevant boundary-layer equation by linearization of the Eqs. (7) and (8) about the boundary values $\hat{y}(0), \hat{T}(0)$, of the equilibrium fields $\hat{y}(x), \hat{T}(x)$, at $x=0$, to yield a set of tractable linear differential equations. The linearization technique is straightforward and involves essentially the same type of algebra used in the linear theory of Brian and Reid [3]. Without dwelling on the algebraic detail here, we simply state the main result of this process, which in effect provides a necessary boundary value $\hat{T}(0)$ on the equilibrium temperature field at $x=0$ in terms of the wall heat flux, as:

$$
\hat{T}(0)=T_{w}-q_{w} / \hat{h},
$$

where $\hat{h}$ is an effective heat transfer coefficient for the reactive boundary layer.

For the example treated by B \& B, where the rate constant $k_{R}$ is independent of temperature and the rate constant $k_{F}$ has the Arrhenius form, one finds after some rather lengthy algebra that $\hat{h}$ is given in terms of a dimensionless parameter $\alpha$ by

$$
\frac{k}{\hat{h} L}=\alpha \equiv(\hat{\eta}-1)\left(\frac{\xi_{0}}{\hat{\xi}}\right) \sqrt{ }\left[\left(\frac{1-\hat{\xi}}{1-\xi_{0}}\right)\left(\frac{2+\hat{\xi}}{2+\xi_{0}}\right)^{3} \frac{1}{m \hat{\eta}^{3}}\right]
$$

where

$$
\hat{\eta}-1=(\eta-1)\left(\frac{T_{0}}{\hat{T}}\right)^{2}\left[\frac{\hat{\xi}(1-\hat{\xi})}{\xi_{0}\left(1-\xi_{0}\right)}\right]
$$

Here, $m$ is the Damköhler number defined in (11) and $\eta$ is the dimensionless constant parameter introduced by B \& B. The quantities $\hat{T}, \hat{\xi}$ refer to the boundary values $\hat{T}(0), \hat{\xi}(0)$ of the outer equilibrium field, from which it is evident that (14) and hence (15) depend in a non-linear way on these boundary conditions.

In the case of complete reaction equilibrium, where $m \rightarrow \infty$ and $\hat{h} \rightarrow \infty$, one has that $\hat{T}(0) \rightarrow T_{w}$ from (13), which corresponds to the standard equilibrium theory. Otherwise, one must solve Eqs. (8) and (12) for $\hat{y}(x), \hat{T}(x)$ subject to the boundary conditions (10) at $x=L$ and (13) at $x=0$. In principle, the value thus obtained for $q_{w}$ should be accurate to terms $0\left(m^{-1 / 2}\right)$ for $m \rightarrow \infty$; whereas, in practice this asymptotic technique for flux is found to give accurate results well into the near-diffusion 
regime $(m \rightarrow 0)$ for the corresponding problem of facilitated mass transport $[7,8,11]$.

For purposes of illustration and comparison here, we shall adopt the assumption of B \& B that the transport coefficients $k$ and $C D$ and the heat of reaction in (8) are constants independent of temperature. In this case, Eq. (8) can be integrated to give a flux relation for the outer equilibrium fields:

$$
q_{w}=\frac{k}{L}\left(\hat{T}-T_{0}\right)-\frac{2 C D \Delta H}{L} \ln \left(\frac{2+\rho}{2+y_{0}}\right),
$$

in addition to the reaction equilibrium condition

$$
\left(\frac{1-\hat{y}}{1-y_{0}}\right)^{3}\left(\frac{y_{0}}{\hat{y}}\right)^{2}=\operatorname{cxp}\left\{\frac{-2 \Delta H}{R}\left(\begin{array}{l}
1 \\
\hat{T}
\end{array}-\frac{1}{T_{0}}\right)\right\} .
$$

Equations (13) (17) then provide sufficient conditions for determination of the unknowns $q_{w}, \hat{T} \equiv$ $\hat{T}(0)$ and $\hat{y} \equiv \hat{y}(0)$.

To summarize, then, in terms of dimensionless variables of the type used in B \& B, Eqs. (13), (16) and (17) can be rewritten respectively as

$$
\begin{gathered}
\hat{\theta}=1-\alpha \phi \\
\phi=\hat{\theta}+\frac{6(\eta-1)}{\lambda \xi_{0}\left(1-\xi_{0}\right)} \ln \left(\frac{2+\hat{\xi}}{2+\xi_{0}}\right)
\end{gathered}
$$

and

$$
\left(\frac{\hat{\xi}}{\xi_{0}}\right)^{3}\left(\frac{1-\xi_{0}}{1-\hat{\xi}}\right)^{2}\left(\frac{2+\xi_{0}}{2+\hat{\xi}}\right)=\exp \left(\frac{\lambda \hat{\theta}}{1+\lambda \mu \hat{\theta}}\right)
$$

where

$$
\begin{aligned}
& \hat{\theta}=\frac{\hat{T}(0)-T_{0}}{T_{w}-T_{0}}, \quad \phi=\frac{q_{w} L}{k\left(T_{w}-T_{0}\right)} \\
& \lambda=\frac{2 \Delta H\left(T_{w}-T_{0}\right)}{R T_{0}^{2}}, \quad \mu=\frac{R T_{0}}{2 \Delta H} .
\end{aligned}
$$

The variable $\hat{\theta}$ is the fractional temperature change across the reaction layer and the quantity $\phi$, which is of primary interest here, represents the reactive 'enhancement' factor for heat flux. Equations (14), (15) and (18) to (20) for $\hat{\xi}, \hat{\theta}, \phi$ represent a complete, implicit algebraic set, which can be solved numerically, once the parameter values $m, \eta, \lambda, \mu$ and the bulk dissociation $\xi_{0}$ are specified.

\section{NUMERICAL RESULTS AND DISCUSSIONS}

The Eqs. (14), (15), (18)-(20) were programmed for an iterative solution on the IBM $360 / 67$ and solutions were performed for parameter values including and beyond those considered in B \& B. In particular, we have also considered the cooling problem not treated in B \& B, where (with an endothermic reaction the parameter $\lambda<0$, and have investigated larger absolute values of $\lambda$ than those of B \& B. Our first objective was to test the present calculations against their finite-difference results. Also we wished to compare the present method with the modified linear approximation of B \& B, namely,

$$
\left.\begin{array}{c}
\phi=\frac{Z \phi_{x}}{\phi_{x}+Z-1} \\
Z=\frac{V\left(\bar{m} \phi_{\infty}\right)}{\tanh \sqrt{\left(\bar{m} \phi_{\infty}\right)}} \\
\bar{m}=m \exp \left(\frac{\lambda}{2+\lambda \mu}\right)
\end{array}\right\}
$$

Here, $\phi_{x}$ is the (exact) equilibrium enhancement obtained by setting $\alpha \equiv 0$ in (14) to (20).

Equation (23) is a modification of the exact linear theory for the regime of weakly-perturbed films, $\lambda \rightarrow 0$, in which, incidentally, $\phi_{\infty} \rightarrow \eta$. As an approximation, (23) has the merit of always giving the correct asymptotes for $m \rightarrow 0$ ('frozen') and $m \rightarrow \infty$ ('equilibrium'). For the parameter values explored by $B$ \& $B$ the formula (23) reportedly gave close agreement (usually to within \pm 4 per cent) with their finite-difference numerical solutions, leading them to conclude that it should be generally valid.

Indeed, Fig. 2 gives a comparison of their modified-linear approximation (ML) with the present boundary-layer method (BL), as a function of Damköhler number, and the results are seen to agree well for $\lambda=2$, where the ML was shown by $B$ $\& B$ to agree with their finite-difference solution. (Accordingly, we have not bothered to try obtaining the precise numbers computed by the latter method from the graphs of B \& B, but have merely recomputed values from the ML formula in (23) and the appropriate values of $\phi_{*}$.) However, the disagreement of the ML and the BL methods for the case of cooling, $\lambda=-2$, is more pronounced, and this is further accentuated for larger $|\lambda|$, as shown in Fig. 3 , where discrepancies as large as $\mathbf{3 0}$ per cent are apparent.

Figures 4 and 5 show the corresponding calculations for the dependence of $\phi$ on the bulk dissociation $\xi_{0}$, extended over a much broader range than that considered in B \& $\mathrm{B}$. The trend toward disagreement between the $\mathrm{ML}$ and $\mathrm{BL}$ methods occurs 
at the larger overall concentration gradients (corresponding to small $\xi_{0}$ for heating and large $\xi_{0}$ for cooling), regions not treated by B \& B.

The rather large discrepancies in the results of the two methods, therefore, appear to be more pronounced as gradients across the reaction-layer become more steep. In this respect, the differences between the cases of heating and cooling are manifest by the temperature 'jump' $1-\hat{\theta}$ across the

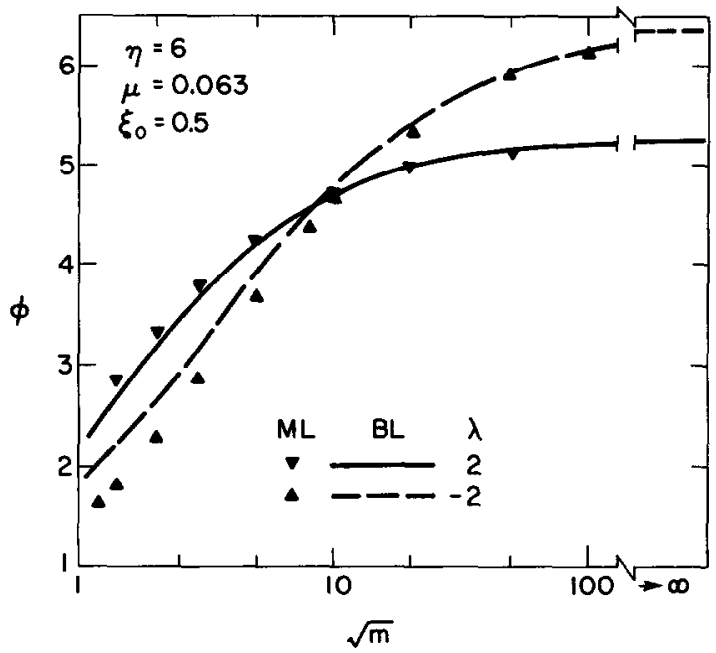

Fig. 2. Enthalpy facilitation factor $\phi$ as a function of Damköhler number $(m)$. Comparison of 'boundary-layer' (BL) and 'modified-linear' (ML) methods of analysis. Moderate values of $\xi_{0}$ and $\lambda$.

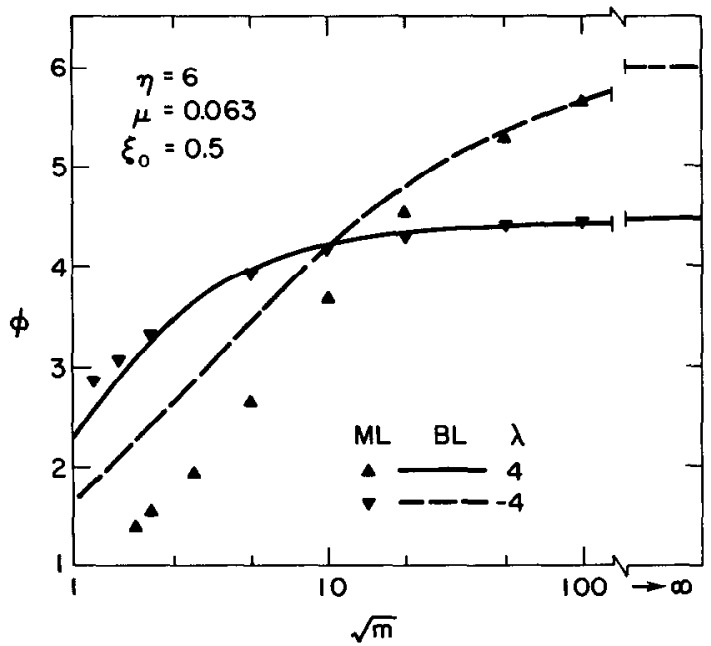

Fig. 3. Enthalpy facilitation factor $\phi$ as a function of Damköhler number $(m)$. Comparison of 'boundary-layer' (BL) and 'modified-linear' (ML) methods of analysis, for moderate values of $\xi_{0}$ and high values of $\lambda$.

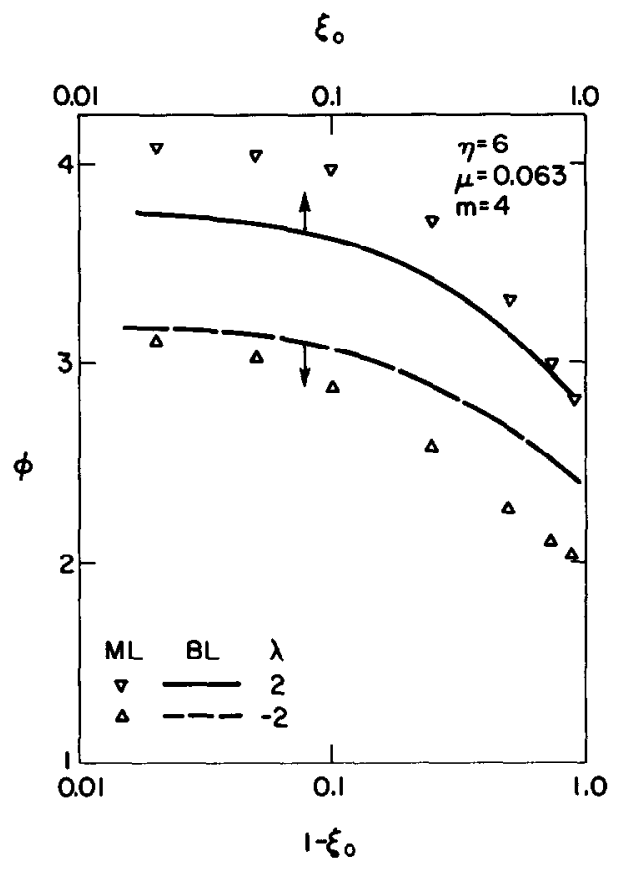

Fig. 4. Effect of degree of dissociation in bulk, $\xi_{0}$, on the facilitation of enthalpy transport at moderate values of $\lambda$. For $\lambda>0(<0), \xi_{0} \rightarrow 0$ (1) represents large driving force in $\xi$.

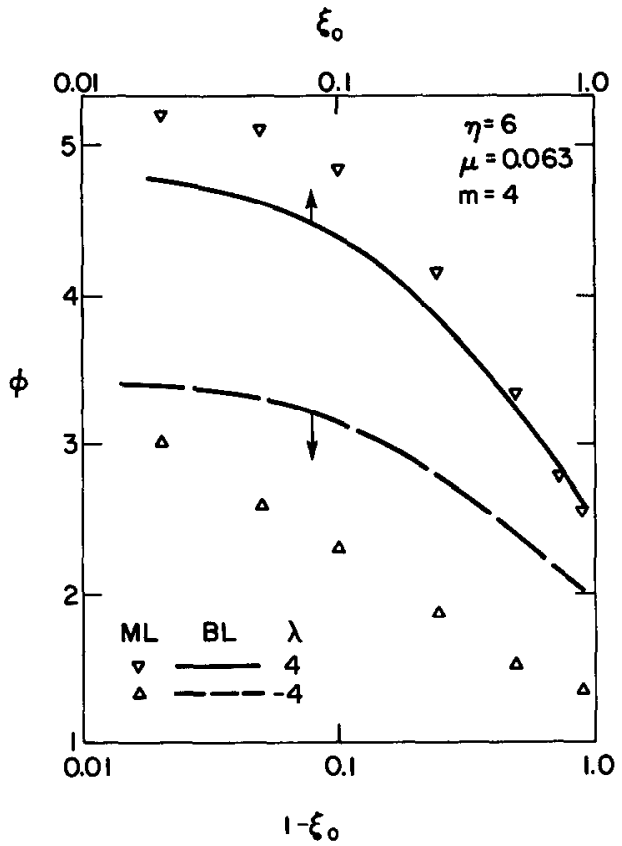

Fig. 5. Effect of degree of dissociation in bulk, $\xi_{0}$, on the facilitation of enthalpy transport at high values of $\lambda$. 


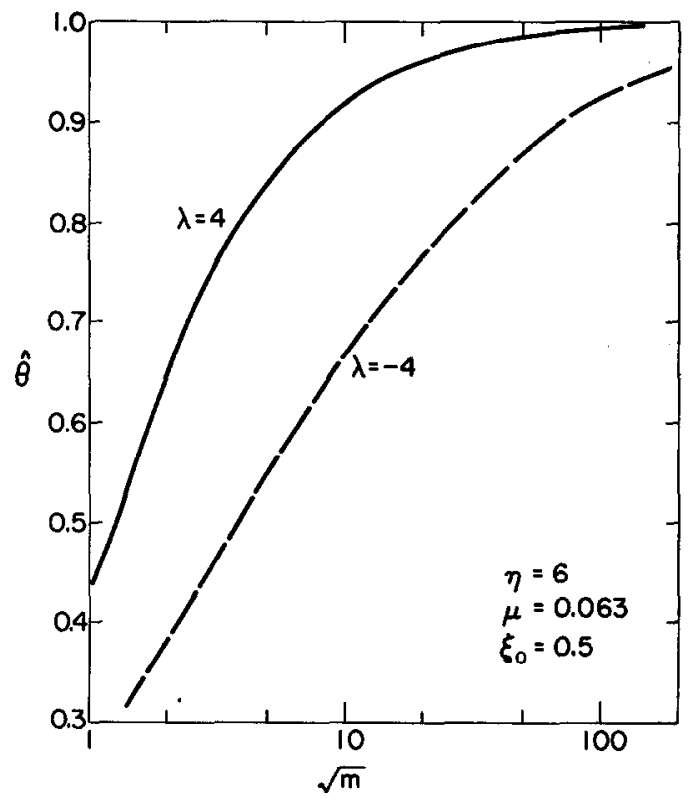

Fig. 6. Dimensionless equilibrium temperature at the wall, $\hat{\theta}$, as a function of Damköhler number $(m)$ at $\xi_{0}=0.5$ and $|\lambda|=4$. The quantity $1-\hat{\theta}$ represents the temperature discontinuity across the reaction boundary layer.

reaction boundary-layer, corresponding to departure from equilibrium, as illustrated by the $\mathrm{BL}$ calculations shown in Fig. 6 for $|\lambda|=4$.

The discrepancies between methods would seem to indicate the desirability of further consideration of the regimes involved, which would necessitate rather laborious finite-difference solutions of (7) to (10). Short of actually attempting this here, we would merely observe that the BL method has been found to give generally accurate results in several related calculations of facilitated mass transport $[7,8,12]$, which tends to give us some confidence in the present type of application.

Acknowledgments-This work was supported in part by Public Health Service Grant GM15152 and a Research Career Development Award (1-K4-GM-8271) to J. S. Schultz. The Authors would like to thank Professor Dave Mason of Stanford University for suggesting this field of application some time ago to one of us (J.D.G.).

\section{NOTATION}
$A$ component $A$
$B$ component $B$
$C$ total concentration, $\mathrm{mol} / \mathrm{cm}^{3}$
$D$ binary diffusion coefficient for components $A$ and $B, \mathrm{~cm}^{2} / \mathrm{sec}$

$\Delta H$ enthalpy of reaction per mol of $A$ reacted, $\mathrm{cal} / \mathrm{mol} A$, one-half activation enthalpy of the forward reaction (1) herein

$\hat{h}$ heat transfer coefficient for reactive boundary layer, $\mathrm{cal} / \mathrm{cm}^{2} \mathrm{sec}^{\circ} \mathrm{K}$

$k$ frozen thermal conductivity $\mathrm{cal} / \mathrm{sec} \mathrm{cm}{ }^{\circ} \mathrm{K}$

$k_{F}$ rate constant for forward reaction, $\mathrm{mol} / \mathrm{sec}$ $\mathrm{cm}^{3}$ atm, Eq. (1)

$k_{R}$ rate constant for reverse reaction, $\mathrm{mol} / \mathrm{sec} \mathrm{cm}^{3}$ $\mathrm{atm}^{3}$, Eq. (1)

$L$ film thickness, $c m$ ( $x_{F}$ in B \& B)

$m^{+} \frac{k_{F 0} p^{2} y_{0} L^{2}}{C_{0} D_{0}}$

$m m^{+} \frac{3}{\xi_{0}(1-\xi)}$

$\bar{m}$ modified (average) value of $m$ in film

$N$ Molar flux, $\mathrm{mol} / \mathrm{cm}^{2} \mathrm{sec}$

$p$ pressure, atm

$q_{w}$ heat flux at wall (positive when heat flows to the gas), $\mathrm{cal} / \mathrm{sec} \mathrm{cm}^{2}$

$r$ reaction rate, $\mathrm{mol} / \mathrm{sec} \mathrm{cm}^{3}$

$\boldsymbol{R}$ universal gas constant, $\mathrm{cal} / \mathrm{mol}{ }^{\circ} \mathrm{K}$

$T$ temperature, ${ }^{\circ} \mathrm{K}$

$x$ distance through film measured from wall, $\mathrm{cm}$

$y$ mole fraction of Component $A$

$Z$ parameter in Eq. (23)

Greek symbols

$$
\begin{aligned}
& \eta^{+} 1+\frac{2 \Delta H^{2}}{R T_{0}^{2}} \frac{C_{0} D_{0}}{k} \\
& \eta 1+\left(\eta^{+}-1\right) \frac{\xi_{0}\left(1-\xi_{0}\right)}{3} \\
& \theta \frac{T-T_{0}}{T_{w}-T_{0}} \\
& \lambda \frac{2 \Delta H\left(T_{w}-T_{0}\right)}{R T_{0}^{2}} \\
& \mu \frac{R T_{0}}{2 \Delta H} \\
& \xi \text { degree of dissociation of component } A= \\
& \frac{1-y}{1+y / 2} \\
& \phi \frac{q_{w} L}{k\left(T_{w}-T_{0}\right)} \\
& \phi_{\infty} \text { value of } \phi \text { for } m \rightarrow \infty
\end{aligned}
$$




\section{REFERENCES}

[1] Brian P. L. T. and Bodman S. W., I. \& E. C. Fundamentals 19643339.

[2] Hirshfelder J. O., J. chem. Phys. 195726274.

[3] Brian P. L. T. and Reid R. C., A.I.Ch.E. Jl 19628322.

[4] Bodman S. W., Brian P. L. T. and Chang T. C., A.I.Ch.E. Jl 1971171304.

[5] Fan S. S. T. and Mason D. M. J. Am. Rocket Soc. 1962 32899.

[6] Kreuzer F. and Hoofd L. J. C., Respir. Physiol. 1972 15104.

[7] Smith K. A., Meldon J. II. and Colton C. K., A.I.Ch.E. Jl 197319102 .

[8] Schultz J. S., Goddard J. D. and Suchdeo S. R., A.I.Ch.E. Jl 197420 Part I. 417, and Part II (in press).
[9] DeGroot S. R. and Mazur P., Non-Equilibrium Thermodynamics. North-Holland, Amsterdam 1962.

[10] Friedlander S. K. and Keller K. H., Chem. Engng Sci. 196520121.

[11] Suchdeo S. R., Goddard J. D. and Schultz. J. S., Proc. Int. Symposium on Oxygen Transport to Tissue. In Advances in Experimental Medicine and Biology, Vol. 37B p. 951 (Eds. D. F. Bruley and H. I. Bicher), Plenum Press, New York 1973.

[12] Suchdeo S. R. and Schultz J. S., Proc. Int. Symposium on Oxygen Transport to Tissue. In Advances in Experimental Medicine and Biology, Vol. 37B p. 969 (Eds. D. F. Bruley and H. I. Bicher), Plenum Press, New York 1973. 